Pak. j. sci. ind. res. Ser. A: phys. sci. 2016 59(2) 114-117

\title{
Effect of Different Processing Stages on the Crystallinity $\%$ and Tensile Strength of $100 \%$ Cotton Fabric
}

\author{
Zahid Hussaina, Muhammad Qamar Tusief ${ }^{\mathrm{b} *}$, Sharjeel Abid ${ }^{\mathrm{c}}$, \\ Muhammad Tauseef Khawer ${ }^{a}$, Nabeel Amin ${ }^{d}$ and Mudassar Abbas ${ }^{d}$ \\ ${ }^{a}$ Interloop (Pvt) Limited Khurrianwala-Jaranwala Road, Faisalabad, Pakistan \\ ${ }^{\mathbf{b}}$ Department of Fibre \& Textile Technology, University of Agriculture, Faisalabad, Pakistan \\ 'Advance Textile Material Engineering, National Textile University, Faisalabad, Pakistan \\ ${ }^{d}$ School of Textile and Design, University of Management and Technology Lahore, Pakistan
}

(received August 31, 2015; revised October 14, 2015; accepted October 15, 2015)

\begin{abstract}
In this study, $100 \%$ cotton fabric was used to check the impact on fabric crystallinity and tensile strength at different processing stages. Desizing, scouring, bleaching, mercerization and resin (only resin $\&$ resin+softener) application were the processes performed on the fabric. X-Ray diffractometer and tensile strength tester were used to determine the crystallinity index (CI) and tensile strength, respectively. Results revealed that from scouring to mercerization crystallinity $(\mathrm{CI})$ decreased while desizing and resin application treatments showed no significant impact on the crystallinity (CI). In case of tensile strength, a decreasing trend from desizing to resin application was observed.
\end{abstract}

Keywords: cotton fabric, tensile strength, crystallinity index, X-ray diffraction

\section{Introduction}

Cotton is mostly used natural cellulosic fibre in textile products. Cellulose has crystalline and amorphous regions. In crystalline region, atoms are arranged in a close packing. There exist three dimensional arrangements due to hydrogen bonding between them. On the other hand in amorphous region, atoms are randomly arranged because of the absence of hydrogen bonding. Number of sites (hydroxyl groups) available in amorphous region governs the bonding or reactivity in suitable conditions for modification in performance properties of cotton fibre, while a material with greater crystallinity percentage has greater strength and a big influence on hardness, density, transparency and diffusion of the material e.g. cotton fibre or fabric (Parikh, 2007).

During different chemical treatments on cotton fibre or fabric, the available sites in the amorphous region react and get modified. Such modifications have impact on the crystallinity and strength of the cotton fibre or fabric. Much research work has been carried out to measure the crystallinity of cotton by using X-ray diffraction method. The crystallite orientation of cotton cellulose was analysed by using improved method of X-ray diffraction spectrometer (Creely et al., 1956). In an other research two types of cotton were treated with anhydrous ethylamine, diethylamine, pyridine and aqueous solutions of sodium hydroxide $(30 \%)$ and *Author for correspondence; E-mail:qamartosief@yahoo.com potassium hydroxide (40\%) for $15 \mathrm{~min}$ to $50 \mathrm{~h}$ to check the impact on moisture regain tensile, mechanical, swelling and optical properties. It was concluded that anhydrous ethylamine, $\mathrm{NaOH}$ and $\mathrm{KOH}$ reduced the crystallinity significantly, while diethylamine and pyridine did not produce any significant change in crystallinity of cotton (Pandey and Iyengar, 1969a). The chemical modification of cotton fibre was also analysed by applying different concentrations of the chemica and their impact on different properties of cotton fibre were observed and concluded that ethylamine, EDA, $\mathrm{KOH}$ and $\mathrm{LiOH}$ reduced the crystallinity of cotton by increasing the concentration (Pandey and Iyengar, 1969b). Cotton fibre and fabric treated with butanetetracarboxylic acid (BTCA) with the catalyst sodium hypophosphite at different concentrations. In the same line the cotton fibre treated with BTCA reduced its crystallinity and strength at different concentrations (Xu, 2003). Similarly, the impact of crosslinking and bleaching treatment on crystallinity of cotton fibres was determined by using formaldehyde and dimethyloldihydroxy ethylene urea (DMDHEU) or citric acid (CA) as cross linking agents for bleached cotton fabric (Parikh, 2007). While in another study the cotton was treated with neutralized vegetable oil base macromolecular crosslinkers to check the impact on cotton crystallinity by using X-ray diffraction methodology (Ford etal., 2010). 
All the above mentioned studies reported on one specific process treatment (surface modification) not on the complete processing stages (from grey fabric to finish) to analyse the gradual decreasing or increasing trend of crystallinity which directly relates with the strength of cotton fabric. The aim of the present study is to analyse the impact of desizing, scouring, bleaching, mercerization followed by the resin application in two ways (one is only resin, while the other is resin along with softener) on the fabric crystallinity and tensile strength.

\section{Materials and Methods}

For this study, $100 \%$ cotton fabric of $110 * 110 / 60 * 60$ was used and analysed in reference to crystallinity and tensile strength by using X-ray diffractometer and tensile strength tester. Lab scale jigger machine was used to perform the experimental work on fabric. Five chemical treatments were applied from desizing to resin application. For desizing, $\alpha$-amylase $5 \%$ on the weight of fabric BEISOL SED (CHT), $2 \mathrm{~mL} / \mathrm{L}$ BEIXON Q (Biodegradable sequesting agent, CHT) and $2 \mathrm{~g} / \mathrm{L}$ FELOSAN FOX (universal detergent with high washing efficiency, CHT) were used for desizing treatment at 90-95 ${ }^{\circ} \mathrm{C}$ for $30 \mathrm{~min}$ followed by one hot wash and then cold wash.

In caustic scouring treatment, $20 \mathrm{~g} / \mathrm{L} \mathrm{NaOH}$ flakes (analytical grade), $1 \mathrm{~g} / \mathrm{L}$ HEPTOL BNF (sequesting agent for strong alkaline treatment, CHT) and $2 \mathrm{~g} / \mathrm{L}$ FELOSAN FOX (universal detergent with high washing efficiency, CHT) were applied at $90-95{ }^{\circ} \mathrm{C}$ for $60 \mathrm{~min}$ followed by $10 \mathrm{~min}$ hot wash and then cold wash. After this, $2 \mathrm{~g} / \mathrm{L}$ acetic acid was used to neutralize the fabric.

In bleaching process, $6 \mathrm{~g} / \mathrm{L}$ hydrogen peroxide $50 \%$ (commercial grade by Sitara peroxide), $2 \mathrm{~g} / \mathrm{L} \mathrm{NaOH}$ flakes, $5 \mathrm{~g} / \mathrm{LCONTAVAN} \mathrm{ICE} \mathrm{(stabilizer,} \mathrm{CHT),} 1 \mathrm{~g} / \mathrm{L}$ HAPTOL BNF and $3 \mathrm{~g} / \mathrm{L}$ FELOSAN FOX were used at $90{ }^{\circ} \mathrm{C}$ for $60 \mathrm{~min}$ followed by hot wash and then cold wash followed by neutralization with $2 \mathrm{~g} / \mathrm{L}$ acetic acid.

In mercerization process, fabric was treated with $30 \%$ solution of caustic soda was used for $60 \mathrm{sec}$ under tension and then hot washed. After this, $2 \mathrm{~g} / \mathrm{L}$ acetic acid used to neutralize the fabric followed by cold wash.

Two different methods were used for the resin application. In first method (only resin application), $30 \mathrm{~g} / \mathrm{L}$ Reaknit EC ( modified DMDHEU, CHT) and $20 \mathrm{~g} / \mathrm{L}$ magnesium chloride (catalyst) were used at 5-6 pH at normal temperature. Fabric was padded at $70 \%$ pick up, dried at $120^{\circ} \mathrm{C}$ for $1 \mathrm{~min}$ and cured at $150{ }^{\circ} \mathrm{C}$ for three min. In the second method (resin+ softener), $30 \mathrm{~g} / \mathrm{L}$ Reaknit EC (DMDHEU, CHT), $30 \mathrm{~g} / \mathrm{L}$ Tubingal SMF-L (microemulsion of silicone, $\mathrm{CHT}$ ) and $20 \mathrm{~g} / \mathrm{L}$ magnesium chloride were used at 5-6 $\mathrm{pH}$ at normal temperature. Fabric was padded at $70 \%$ pick up, dried at $120^{\circ} \mathrm{C}$ for $1 \mathrm{~min}$ and then cured at $150{ }^{\circ} \mathrm{C}$ for $3 \mathrm{~min}$.

All the above mentioned chemicals were commercial grade of CHT chemical company and sourced from a reputed textile mills Khurrianwala, Faisalabad, Pakistan Caustic soda, magnesium chloride and acetic acid were used of analytic grade.

X-ray diffractometer (Model Xpert Pro Pan Analytical) was used to determine the crystallinity index (CI). In $\mathrm{X}$-ray diffraction, X-rays beams were incident on the sample diffracted a beam whose angular dependence was measured by photomultiplier detector travelling upon a goniometer in circle and provide a graph of intensity (counts) of X-ray photons detected as a function of $2 \theta$ (the angle between the detector position and the direction of incident beam) as shown in Fig. 1.

From the Fig. 2, crystallinity (CI) can be measured by the formula.

$$
\text { Crystallinity } \%=[(\mathrm{Ic}-\mathrm{Iam}) / \mathrm{Ic}] * 100
$$

where:

Ic $=$ the intensity of the principal cellulose peak at $2 \theta=$ $22.7^{\circ}$,

Iam $=$ the intensity of amorphous peak at $2 \theta=18^{\circ}$.

Tensile strength tester Lloyd of Ametek Company was used to check the impact on warp wise and weft wise strength of the cotton fabric after every process.

\section{Results and Discussion}

Grey fabric. The values related to the crystallinity and tensile strength (warp wise and weft wise) of the grey fabric (Sample 1) are mentioned in Table 1. These results reveal a decreasing trend in the crystallinity and tensile strength of the fabric.

Desized fabric. The effect of desizing process on fabric in respect of its crystallinity and tensile strength was determined. The results given in Table 1 and further illustrated in Fig. 2-3 depict non significant impact on crystallinity $\%$ of the fabric after desizing. But the tensile strength of the fabric reduced from $478.05 \mathrm{~N}$ to 439.03 on warp wise and from $469.7 \mathrm{~N}$ to $433.11 \mathrm{~N}$ on 
Table 1. Results of crystallinity(\%) and tensile strength (warp \& weft wise)

\begin{tabular}{lllll}
\hline \hline $\begin{array}{l}\text { Sample } \\
\text { No }\end{array}$ & Process steps & $\begin{array}{c}\text { Crystallinity } \\
(\%)\end{array}$ & $\begin{array}{l}\text { Tensile } \\
\text { strength (N) } \\
\text { warp wise }\end{array}$ & $\begin{array}{l}\text { Tensile } \\
\text { strength (N) } \\
\text { weft wise }\end{array}$ \\
\hline 1 & Grey & 68.32 & 478.05 & 469.7 \\
2 & Desize & 68.11 & 439.03 & 433.11 \\
3 & Scoured & 67.23 & 419.03 & 412.4 \\
4 & Bleached & 62.47 & 362.71 & 353.56 \\
5 & Mercerized & 61.56 & 366.89 & 352.89 \\
6 A & Resin & 64.77 & 247.08 & 226.09 \\
6 B & Resin+Softener 62.05 & 279.36 & 254.6 \\
\hline \hline
\end{tabular}

weft wise. Size material applies on yarn level before weaving to provide adequate strength for the next process which helps during the weaving process. But during desizing size material removed from the fabric that affects the strength of the fabric. Both these factors reduce the strength after desizing in fabric form.

Scoured fabric. Both crystallinity and tensile strength decreased after caustic scouring as shown in Table 1 and Fig. 2-4. During this process, natural impurities of cotton removed by the reaction of caustic soda at high temperature. Chemical degradation and the effect of elevated temperature reduced the crystallinity from $68.11 \%$ to $67.23 \%$ and tensile strength about $20 \mathrm{~N}$.
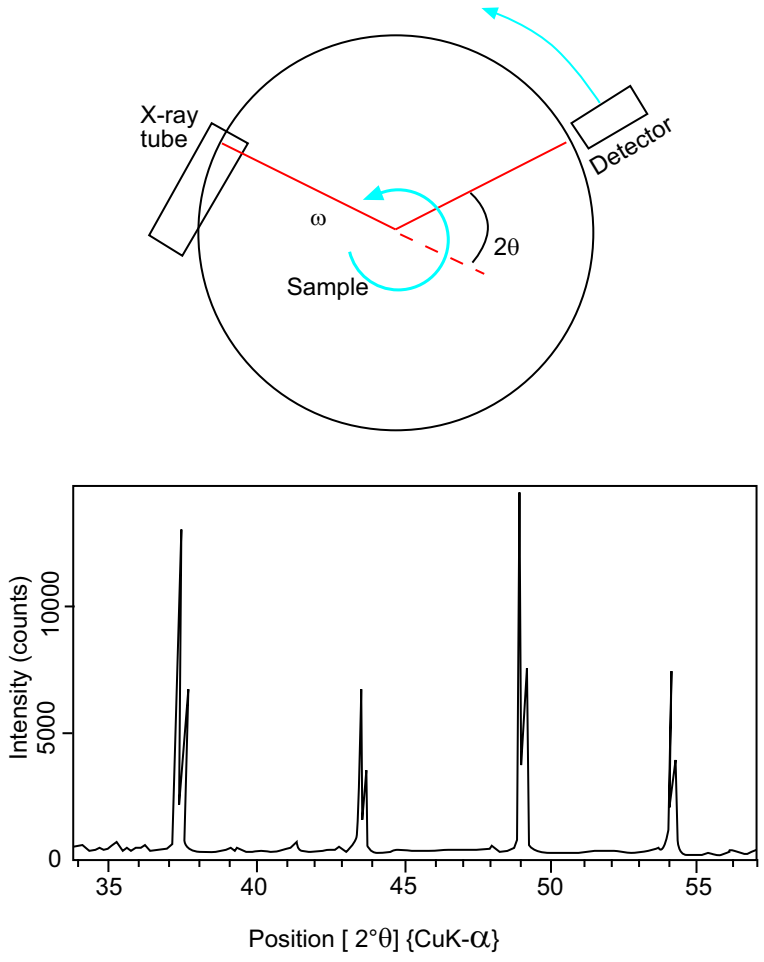

Fig. 1. Shows the working principle of X-Ray diffractometer and graphical output.
Bleached fabric. Significant decrease in crystallinity and tensile strength was observed after the bleaching process as shown in Table 1 and Fig. 2-4. During bleaching process, natural and colour impurities removed from the fabric. Hydrogen bonding from the crystalline region breaks due to the chemical degradation of the fabric which reduced the crystallinity from $67.23 \%$ to $62.47 \%$ and also the tensile strength reduced significantly.

Mercerized fabric. During mercerization caustic soda reacts with the cellulose and breaks down the hydrogen bonding of the atoms in crystalline region of the cotton fibre. This provides greater number of sites for reaction. Ultimately the pick up of the cotton fabric enhanced (El Badry et al., 2013). Due to this crystallinity of the

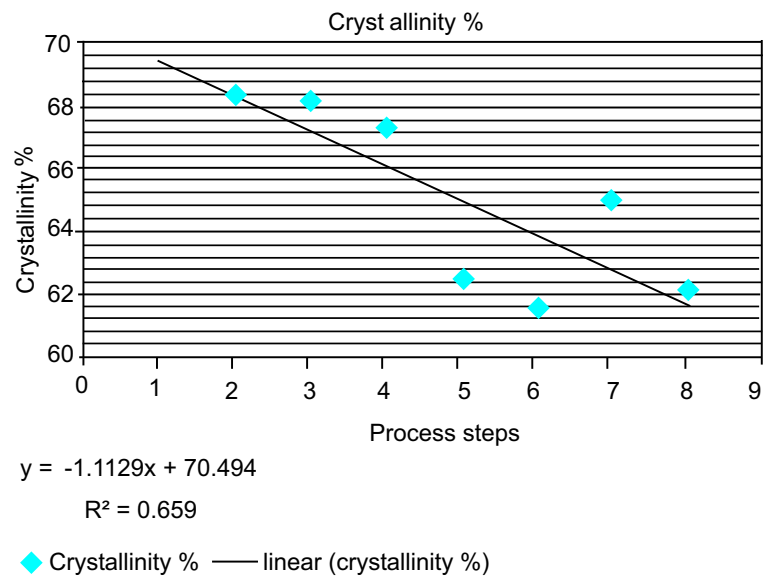

Fig. 2. Graphical explanation of crystallinity at different processing steps.

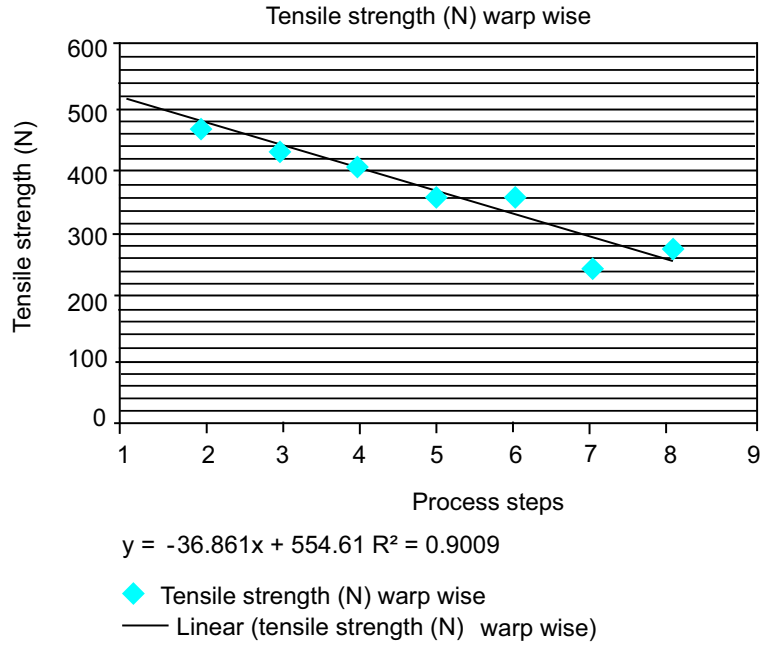

Fig. 3. Graphical explanation of warp wise tensile strength $(\mathrm{N})$ at different processing steps. 


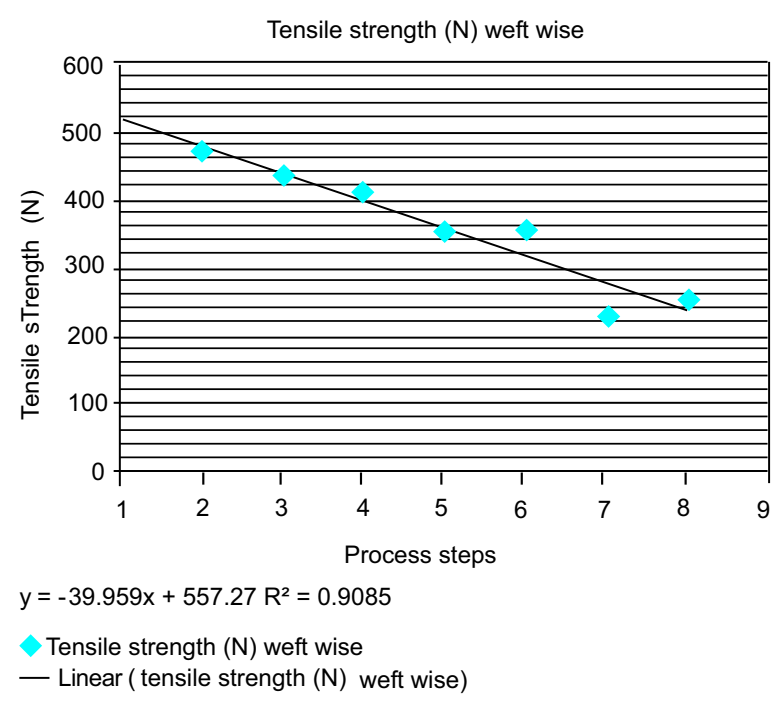

Fig. 4. Graphical explanation of weft wise tensile strength $(\mathrm{N})$ at different processing steps.

fabric decreased slightly from $62.47 \%$ to $61.56 \%$ in the mercerized sample while the warp wise strength of the fabric increased due to parallelization of the fibre along vertical axis under tension as shown in Table 1 and Fig. 2-4.

Resin finished fabric. After the resin application crystallinity $\%$ of the fabric increased significantly from 61.56 to 64.77 due to the cross linking of resin finish (DMDHEU). This helps to enhance the bonding and close packing in the crystalline region of the fibre. On the other hand the tensile strength of the fabric reduced while the stiffness of the fabric enhanced by the resin application as shown in Table 1 and Fig. 2-4.

Resin+softener finished fabric. After the application of resin with softener, a slight increase of crystallinity from $61.56 \%$ to $62.05 \%$ was observed in the fabric. Because the effectiveness of resin crosslinking reduced by the combination of softener, crystallinity of the fabric enhanced on less \% age as compared to only resin application as shown in Table 1 and Fig. 2. Fabric (softner+resin) was also soft comparatively from the resin padded fabric. It was due to the softness induced by the micro-emulsion silicon based softener. Resin with softener finish impact on tensile strength was also less as compared to only resin padded fabric due to the flexibility enhanced by softener and less fiber stiffness as shown in Table 1 and Fig. 3-4.

\section{Conclusion}

It is concluded from the above study that, $100 \%$ cotton fabric significantly lose its degree of crystallinity (crystallinity \%) during caustic scouring, bleaching and mercerization due to chemical degradation and elevated temperature impact, while desizing has no significant effect on crystallinity $\%(\mathrm{CI})$. But the resin application increases the crystallinity $\%$ age due to the crosslinking and bonding in the cotton fabric structure. Tensile strength decreases during all the processing stages from desizing to resin finish treatment. But scouring, bleaching and resin application significantly reduces the tensile strength of the fabric.

\section{References}

Creely, J. J., Sega, L. 1., Ziifle, H. M. 1956. Determination of the degree of crystallite orientation in cotton fibers by means of the recording X-Ray diffraction spectrometer. Textile Research Journal, 28: 789795.

El Badry, K., Saleh, S.M., Bahlood, S. O. 2013. effect of mercerization techniques on cotton towels properties. Journal of Applied Sciences Research., 9: 2386.

Ford, E. N. J., Mendon, S. K., Thames, S. F., Rawlins, J. W. 2010. X-ray diffraction of cotton treated with neutralized vegetable oil-based macromolecular crosslinkers. Journal of Engineered Fibres and Fabrics, 5: 10-20.

Pandey, S. N., Iyengar, R. L. N. 1969a. Studies of chemically modified cotton. Part I: Effect of chemical treatments for varying periods on crystallinity and certain other properties of cotton. Textile Research. Journal, 39: 15-23.

Pandey, S. N., Iyengar, R. L. N. 1969b. Studies on chemically modified cotton. Part II: Effect of different concentrations of chemicals on crystallinity and certain other properties of cotton. Textile Research Journal, 39: 24-31.

Parikh, D.V. 2007. X-ray crystallinity of bleached and crosslinked cottons.Textile Research Journal, 77: 612-616.

$\mathrm{Xu}, \mathrm{W} .2003$. Effect of crosslinking treatment on the crystallinity, crystallite size, and strength of cotton fibres.Textile Research Journal, 73: 433-436. 